\title{
Repetitive Motor Practice Impacts Neuromuscular System Plasticity in Healthy and Stroke Populations
}

\section{Crystal L Massie ${ }^{1 *}$, Christina Christenson ${ }^{1}$ and Ana Durand-Sanchez ${ }^{2}$}

${ }^{1}$ Department of Occupational Therapy, School of Health and Rehabilitation Science, Indiana University, Indianapolis, USA

${ }^{2}$ Department of Physical Medicine and Rehabilitation, Baylor College of Medicine, TIRR Memorial Hermann Hospital, Houston, TX, USA

\begin{abstract}
Neuroplasticity is an important factor for upper-extremity stroke rehabilitation, yet the amount of neuromodulation achieved with an acute session of motor practice is not fully characterized. The objective of this study was to determine how engaging in isometric motor practice acutely influences motor control, dexterity, and neuromodulation. Thirteen healthy controls and 5 stroke survivors participated in 2 separate experiments. Assessments [Box and Block Test (BBT), force steadiness, Transcranial Magnetic Stimulation (TMS)] were completed immediately before and following motor practice. Two force steadiness trials were completed at 10 and $20 \%$ of the maximum voluntary contraction (MVC). The TMS outcome included an average of 12 suprathreshold stimulations with motor evoked potentials (MEP) amplitudes recorded. Data were analyzed using paired $t$-tests for each experiment. Both participant groups were able to move significantly more blocks with the hand that practiced, but not with the non-practicing side. Force steadiness and muscle activity significantly improved for both groups, yet the MEP amplitudes did not. These results suggest that a short intervention of isometric wrist extension can influence motor control and dexterity, but may have a slight impact on neuromodulation. This demonstrates the importance of better understanding how possible rehabilitation interventions influence neuroplasticity through neuromodulation.
\end{abstract}

Keywords: Stroke; Motor practice; Transcranial magnetic stimulation; Neuroplasticity; Motor control

\section{Introduction}

Neuroplasticity or the brain's ability to change and reorganize following injury, is a known critical factor to the rehabilitative process following neurological injuries [1]. In addition, neuroplasticity is now being considered as a component that may influence rehabilitation following musculoskeletal injuries [2]. While many non-invasive brain stimulation studies have focused on increasing cortical excitability during a single session [3-8], fewer studies have investigated the impact of repetitive motor practice to induce acute changes in motor performance and cortical excitability [9]. This is especially true regarding function at the wrist, as most of the interventions so far discussed in the literature focus on intrinsic hand muscles.

The ability to induce experience-dependent neuroplasticity is an important contribution to the rehabilitation process $[1,10]$. While traditionally the focus is on neurological injuries such as stroke and traumatic brain injury, recently these concepts are being expanded to musculoskeletal rehabilitation [2]. The argument is that neuroplasticity likely plays a role in the rehabilitation of musculoskeletal conditions such as fractures, cumulative trauma, or chronic pain. Currently, typical treatment options for musculoskeletal injuries focus on regionspecific movement performance without considering the potential for plasticity of the central nervous system [2]. The opportunity to better understand neuromodulation in neurologically-intact populations and with repetitive motor practice is important and will likely contribute to this emerging area for neuroplasticity. The potential impact would be to facilitate improved rehabilitation methods for millions of patients around the world.

Repetitive motor practice has been suggested as a mechanism to induce acute plasticity in the motor cortex. In a seminal study by Classen and colleagues [9], repetitive motor practice induced cortical changes following approximately 15 to $30 \mathrm{~min}$ of repetitive thumb movement in the direction opposite of a transcranial magnetic stimulation (TMS) induced movement. After the training, the direction of TMS evoked movement was in the practiced direction suggesting that the neural representations are plastic and can change quickly with practice. These changes were induced in a matter of minutes, but then also returned to baseline within approximately $20 \mathrm{~min}$, thus demonstrating how quickly experience-dependent plasticity can be elicited but potentially not maintained. These concepts are important to consider within a rehabilitation treatment session because of the potential to maximize the degree of neuroplasticity with repetition. The authors suggest the encoding of a practiced movement may be the initial step in skill acquisition [9]. One of the challenges, however, that remains is the limited number of studies that have investigated the impact of practice on motor performance and cortical excitability. This is especially true considering wrist function, which is often impaired following stroke [11,12]. Lodha and colleagues [12] demonstrated that survivors of stroke are not able to generate as much wrist extension force and have more difficulties maintaining a steady contraction compared to controls. Further, wrist extension is an important criteria for inclusion in intensive rehabilitation studies such as constraint-induced movement therapy [13] which is salient because wrist extension is more difficult to perform post-stroke as compared to wrist flexion.

Most of the research has focused on investigating the impact of noninvasive brain stimulation which has been combined motor practice [7,14-16]. Mirdamadi and colleagues [7] primarily investigated the combined effects of theta burst stimulation with a simultaneous wrist contraction. This study also included a wrist flexion contraction only protocol for comparison. The contraction alone protocol only had a small influence on extrinsic hand muscles. The study, however, did not

*Corresponding author: Crystal L Massie, Assistant Professor, Department of Occupational Therapy, School of Health and Rehabilitation Science, Indiana University, Indianapolis, 1140 W. Michigan St. CF 306 Indianapolis, IN, 46202 USA, Tel: 3172789617; E-mail: Massiec@iu.edu

Received July 25, 2016; Accepted August 10, 2016; Published August 17, 2016

Citation: Massie CL, Christenson C, Durand-Sanchez A (2016) Repetitive Motor Practice Impacts Neuromuscular System Plasticity in Healthy and Stroke Populations. Int J Neurorehabilitation 3: 221. doi:10.4172/2376-0281.1000221

Copyright: ( 2016 Massie CL, et al. This is an open-access article distributed under the terms of the Creative Commons Attribution License, which permits unrestricted use, distribution, and reproduction in any medium, provided the original author and source are credited. 
investigate the impact of the interventions on motor performance. A combined study of cortical changes and motor function following wrist training would be important contribution to the Neurorehabilitation community. As such, the current study was aimed at determining the impact of a repetitive motor practice protocol on neuroplasticity both in a neurologically-intact, healthy population and in a stroke population. Two separate experiments were conducted to determine the neurophysiological response to motor practice. We hypothesized that the repetitive motor practice performed during the study would induce changes in motor performance, as well as in cortical excitability.

\section{Methods}

Experiment one consisted of thirteen healthy participants; the second experiment consisted of 5 stroke subjects. See Table 1 for participant demographics and all provided written informed consent prior to completing the experiments. The visit consisted of an assessment immediately prior to and following a motor training intervention. Motor control assessments included the Box and Block Test (BBT), as well as force steadiness and electromyography during a wrist extension task. The BBT is a measure of dexterity assessed via the number of small blocks moved in a minute. Following the BBT, participant's right hand was positioned in a padded custom wrist device used to measure wrist extension with a force transducer (MLP100, Transducer Techniques). For the $2^{\text {nd }}$ experiment, the stroke-affected arm was tested. Surface electromyography (EMG) was monitored by a pair of surface electrodes for the extensor carpi radialis (ECR) and flexor carpi radialis (FCR) muscles in a bipolar arrangement (AD Instruments). The signal was digitized at $2000 \mathrm{~Hz}$. An isometric maximum voluntary contraction (MVC) was tested over approximately three seconds [17]. Following the MVC, a force steadiness task was conducted with two trials of at least 10 seconds at $10 \%$ and $20 \%$ of the MVC. Participants were asked to increase their force levels by extending into the force transducer to a level of $10 \%$ or $20 \%$ MVC and then asked to hold that as steady as possible for at least 10 secs.

Neurophysiological assessment used TMS to assess cortical excitability. Motor cortex stimulation was delivered with a $70 \mathrm{~mm}$ figure-of-eight shaped coil and two Magstim 2002 stimulators connected through a bi-stimulation module (Magstim Ltd, UK). The stimulation area (hot spot) was determined by moving the coil systematically in $1 \mathrm{~cm}$ movements until the motor evoked potentials (MEP) was optimized (ECR). MEP was measured with the same surface EMG electrodes. Motor threshold was expressed as the lowest magnetic stimulus intensity eliciting MEPs $>50$ microvolts in at least 5 of 10 consecutive stimulations. Cortical excitability was assessed using stimulation at $116 \%$ of resting motor threshold as previously reported in a similar study [6].

Following the assessments, participants completed the intervention. The motor practice (MP) intervention consisted of 30 isometric contractions in the same device for $6 \mathrm{~s}$ followed by a $30 \mathrm{~s}$ rest. A custom software application provided a visual cue to begin the voluntary contraction (light turned on) and visual feedback provided the percentage of EMG produced. The MP intervention required subjects to generate extension muscle activity that exceeded a threshold of $20 \%$ of the maximum EMG activity recorded during MVCs. Subjects were instructed to maintain the contraction at or slightly above $20 \%$ for $6 \mathrm{~s}$ and then asked to relax after the light turned off. Immediately following the practice session, participants completed a post-assessment comprised of force steadiness, TMS and the BBT.

The coefficient of variation $(\mathrm{CV})$ was calculated as a measure of steadiness for each trial of the force steadiness task. The TMS data were calculated as an average of the 12 peak-to-peak amplitudes. Data were then analyzed separately for each group according to each outcome measure using a paired samples $\mathrm{t}$-test. The significance level was set at $\mathrm{p}<0.05$.

\section{Results}

Participants from both experiments significantly increased the number of blocks transported with the hand that completed the MP intervention, but not with the hand that did not practice as illustrated in Figure 1. The healthy group, on average, transported 3 more blocks with the right hand following the intervention compared to the pretest $(\mathrm{p}=0.02)$. In the 2 nd experiment, the stroke group increased the number of blocks by $2.8(\mathrm{p}=0.01)$ with the stroke-affected arm that engaged in the practice. There was no significant difference on the unpracticed and less-affected side.

The steadiness on the force control task significantly improved for both experimental groups. The healthy group had a $20 \%$ improvement on steadiness following the intervention. This was coupled with a significant increase in muscle activity post-intervention by $20 \%$ $(p=0.02)$. Similarly, in the stroke group, the CV of force significantly improved by $41.6 \%(p=0.01)$ coupled with a significant increase in

\begin{tabular}{|c|c|c|c|c|}
\hline \multicolumn{7}{|c|}{ Healthy Control Group $(\mathrm{n}=13)$} \\
\hline \multicolumn{7}{|c|}{$\begin{array}{c}\text { Age } 26.9( \pm 8.8), 6 \text { female } \\
\text { Stroke Group }(\mathrm{n}=5)\end{array}$} \\
\hline Participant & Sex & Age & Months since stroke & Fugl-Meyer \\
\hline 1 & M & 54 & 30 & 53 \\
\hline 2 & M & 53 & 84 & 23 \\
\hline 3 & M & 84 & 18 & 61 \\
\hline 4 & F & 56 & 30 & 31 \\
\hline 5 & M & 68 & 8 & 48 \\
\hline
\end{tabular}

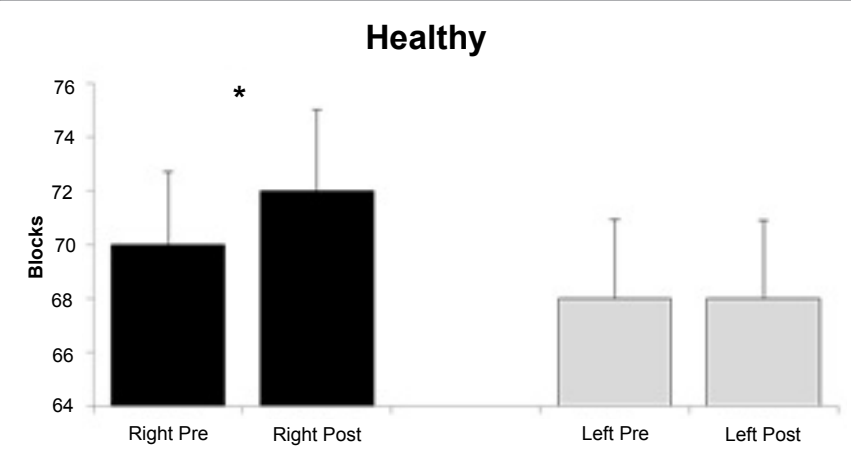

Stroke

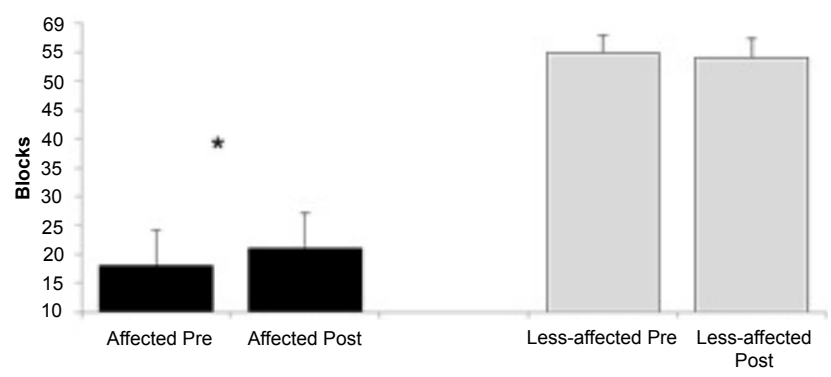

Figure 1: Box and block test (BBT). 

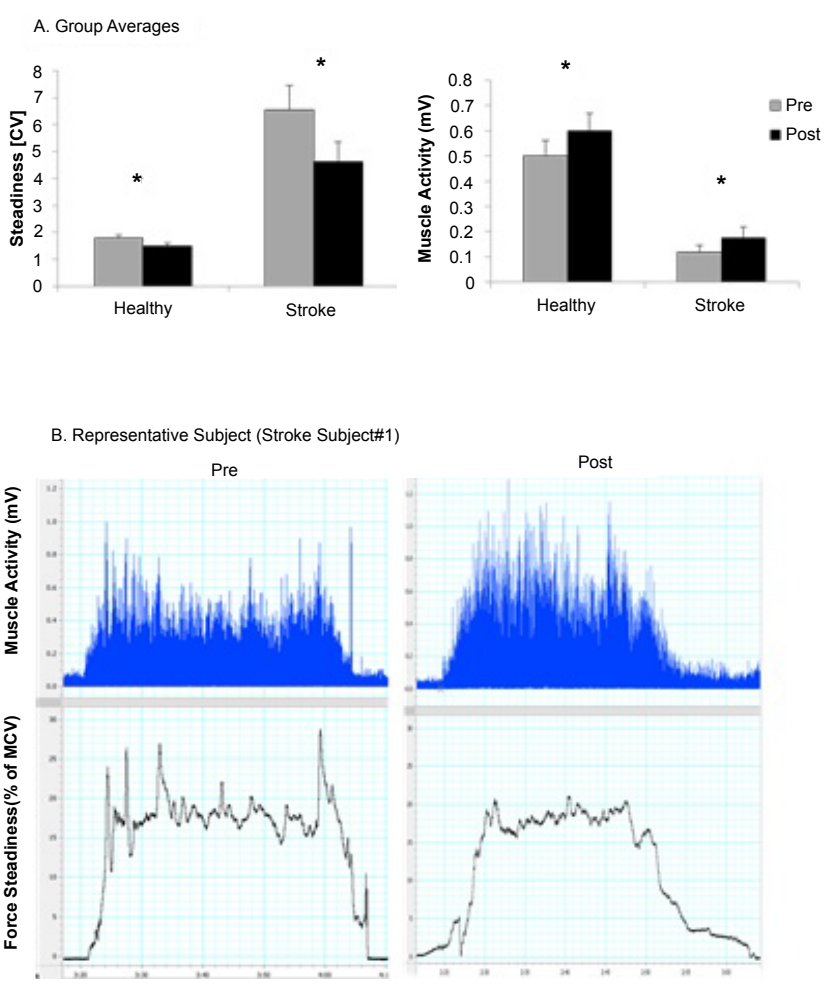

Figure 2: Force steadiness.

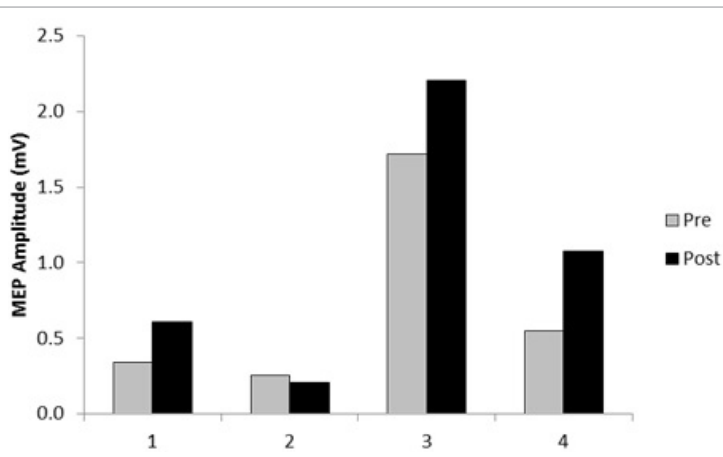

Figure 3: Motor evoked potential data for each stroke participant.

muscle activity levels of the wrist extensor (ECR) by $46.9 \%(p=0.01)$. These results are illustrated in Figure 2 along with representative data from one of the stroke participants.

The cortical excitability data were not significantly different for the healthy control group with pre-test average of $1.8 \mathrm{mV}$ and 2.3 $\mathrm{mV}$ at post $(p=0.2)$. There was a small increase in the MEP amplitude following the intervention in the stroke group, but this failed to reach significance $(p=0.05)$. Individual changes in the MEP amplitude are illustrated in Figure 3 for the 4 stroke participants from whom MEP data were elicited. Only data from 4 of the subjects are presented because TMS responses were not evoked in the other participant.

\section{Discussion}

The results of the two experiments suggest that a short intervention of isometric wrist extension can influence dexterity and motor control in both healthy and stroke populations. Consistencies between both groups were observed in BBT scores of the arm and hand involved with the training, yet the hand that did not practice did not improve its performance. Additionally, both groups demonstrated improved steadiness following the motor practice session with a concomitant increase in the amount of extensor muscle activity. These consistencies between a healthy, neurologically-intact population and a stroke population are encouraging because such information is likely to hasten the translation of neuroplasticity research. The results of this study also support the use of repetitive motor practice as a safe and viable method to elicit changes in motor performance, yet with only modest changes in cortical excitability.

The consistencies between groups are supported by literature, yet are seldom directly compared. Given the group size differences we did not directly compare the stroke group to the healthy control group, but rather observed the directions and patterns of change between the two groups. The consistencies between the two groups were encouraging as Kleim and Jones [1] suggest the processes of neuromodulation in neurologically-intact individuals should be the same as neurologicallycompromised individuals. This supports the translation of principles from 'healthy' to disease-affected populations, and may be used to support larger studies in healthy populations to guide more specific studies in populations with neurological disorders. Further, the healthy 'control' study can also be used to establish a basis for the plasticity potential in individuals who may not have a neurological disorder, but rather a musculoskeletal disorder or condition. The current study used various levels of outcomes including functional dexterity measured by the BBT, motor control measured by force steadiness and muscle output, and cortical excitability. We observed significant changes in dexterity and motor control after a $15 \mathrm{~min}$ intervention which suggests that we did not have a floor effect with these outcome measures. Although the healthy group was much steadier on the wrist extension task at pre-training compared to the stroke group (1.8 compared to 6.6), there was still an improvement in the healthy group after the training. The current study's results are consistent with a previous report [12] of steadiness in the wrist extensors in both the stroke group $(6.5 \%)$ and a healthy group (1.8\%), suggesting that the differences in methods are likely negligible. The current study extends these previous findings by incorporating EMG as an additional outcome. We found that improvements in steadiness were accompanied by an increase in the amount of muscle activity. This is an important finding because previous studies have reported inconsistent findings for studies combining repetitive transcranial magnetic stimulation (rTMS) plus voluntary practice studies to influence motor function $[6,16]$. These inconsistencies, however, are likely due to differences in protocols.

This study informs clinical practice by indicating the degree of neuromodulation attainable with specific, repetitive practice of isometric wrist extension in survivors of stroke and in a healthy control population. This is an important consideration in research studies implementing technologies such as rTMS to influence neuromodulation as an adjunctive treatment for stroke rehabilitation; this study highlights the strength of voluntary practice as a stimulus for neuroplasticity. Although we did not observe significant changes in the amount of cortical excitability following the training, this result is consistent with previous literature [7]. These results may suggest that practice alone does not have sufficient potency to elicit changes in the cortex, however Classen et al. [9] demonstrated directional changes in TMS evoked thumb movements. This variation may be due to a difference in outcomes (evoked movements rather than motor evoked potentials) and is an area for future research. These differences may also have resulted due to the heterogeneity of our stroke subjects given they ranged in age, time since stroke, and stroke-severity. Motor practice 
only protocols should be further evaluated against non-invasive brain stimulation protocols.

In conclusion, this study contributes to the growing body of literature to support repetitive motor practice as a means to elicit neuroplasticity. The consistencies between groups suggest that stroke individuals can respond similarly to a neurologically-intact population. Additionally, this study highlights the potential importance of wrist function and practice on dexterity. The task implemented was a simple isometric extension task, yet we observed changes in dexterity in both groups. Wrist function should not be overlooked with regards to improvements in functional ability and may need to be considered a more isolated target for neurorehabilitation approaches. For example, if an individual with stroke lacks wrist extension and control, this limitation could be hindering motor performance just as much as limited control of the fingers can. We observed one of our stroke participants was not able to transport any blocks prior to the intervention and then was able to transport 4 blocks after the intervention. The participant perceived this as a meaningful improvement and a sign of hope for future changes that could be elicited with targeted practice. A continued research focus on neuroplasticity for improving motor abnormalities and wrist function with both neurological disorders and potentially musculoskeletal disorders is warranted. This focus is consistent with other literature that continues to focus on relating neuroplasticity and cortical excitability in both healthy and neurological populations including vascular related dementia [18].

\section{References}

1. Kleim JA, Jones TA (2008) Principles of experience-dependent neural plasticity: Implications for rehabilitation after brain damage. Journal of Speech Language and Hearing Research 51: S225-S239.

2. Snodgrass SJ, Heneghan NR, Tsao H, Stanwell PT, Rivett DA, et al. (2014) Recognising neuroplasticity in musculoskeletal rehabilitation: A basis for greater collaboration between musculoskeletal and neurological physiotherapists. Manual Therapy 19: 614-617.

3. Fujiwara T, Rothwell JC (2004) The after effects of motor cortex rTMS depend on the state of contraction when rTMS is applied. Clinical Neurophysiology 115 1514-1518.

4. Khedr EM, Rothwell JC, Ahmed MA, Shawky OA, Farouk M, et al. (2007) Modulation of motor cortical excitability following rapid-rate transcranial magnetic stimulation. Clinical Neurophysiology 118: 140-145.

5. Pell GS, Roth Y, Zangen A (2011) Modulation of cortical excitability induced by repetitive transcranial magnetic stimulation: Influence of timing and geometrical parameters and underlying mechanisms. Progress in Neurobiology 93: 59-98.

6. Massie C, Tracy B, Malcolm M (2013) Functional repetitive transcrania magnetic stimulation increases motor cortex excitability in survivors of stroke. Clinical Neurophysiology 124: 371-378.

7. Mirdamadi JL, Suzuki LY, Meehan SK (2015) Agonist contraction during intermittent theta burst stimulation enhances motor cortical plasticity of the wrist flexors. Neuroscience Letters 591: 69-74.

8. Zimerman M, Heise KF, Hoppe J, Cohen LG, Gerloff C, et al. (2012) Modulation of training by single-session transcranial direct current stimulation to the intact motor cortex enhances motor skill acquisition of the paretic hand. Stroke 43: 2185-2191.

9. Classen J, Liepert J, Wise SP, Hallett M, Cohen LG (1998) Rapid plasticity of human cortical movement representation induced by practice. Journal of Neurophysiology 79: 1117-1123.

10. Chen R, Tam A, Bütefisch C, Corwell B, Ziemann U, et al. (1998) Intracortica inhibition and facilitation in different representations of the human motor cortex. Journal of Neurophysiology 80: 2870-2881.

11. Hu X, Tong K, Tsang VS, Song R (2006) Joint-angle-dependent neuromuscula dysfunctions at the wrist in persons after stroke. Archives of Physical Medicine and Rehabilitation 87: 671-679.

12. Lodha N, Naik SK, Coombes SA, Cauraugh JH (2010) Force control and degree of motor impairments in chronic stroke. Clinical Neurophysiology 121: 1952-1961.

13. Massie C, Malcolm MP, Greene D, Thaut M (2009) The effects of constraintinduced therapy on kinematic outcomes and compensatory movement patterns: An exploratory study. Archives of Physical Medicine and Rehabilitation 90: 571-579.

14. Castel-Lacanal E, Gerdelat-Mas A, Marque P, Loubinoux I, SimonettaMoreau M (2007) Induction of cortical plastic changes in wrist muscles by paired associative stimulation in healthy subjects and post-stroke patients. Experimental Brain Research 180: 113-122.

15. Castel-Lacanal E, Marque P, Tardy J, de Boissezon X, Guiraud V, et al. (2009) Induction of cortical plastic changes in wrist muscles by paired associative stimulation in the recovery phase of stroke patients. Neurorehabilitation and Neural Repair 23: 366-372.

16. Todd G, Rogasch NC, Flavel SC, Ridding MC (2009) Voluntary movement and repetitive transcranial magnetic stimulation over human motor cortex. Journal of Applied Physiology 106: 1593-1603.

17. Tracy BL, Maluf KS, Stephenson JL, Hunter SK, Enoka RM (2005) Variability of motor unit discharge and force fluctuations across a range of muscle forces in older adults. Muscle \& Nerve 32: 533-540.

18. Pennisi G, Bella R, Lanza G (2015) Motor cortex plasticity in subcortical ischemic vascular dementia: What can TMS say? Clinical Neurophysiology 126: 851-852. 